\title{
Features of MCNP6
}

\author{
T. Goorley, ${ }^{1}$ M. James, ${ }^{2}$ T. Booth,${ }^{3}$ F. Brown, ${ }^{1}$ J. Bull, ${ }^{1}$ L.J. Cox, ${ }^{1}$ \\ J. Durkee, ${ }^{2}$ J. Elson, ${ }^{2}$ M. Fensin, ${ }^{2}$ R.A. Forster, ${ }^{5}$ J. Hendricks, ${ }^{4}$ \\ H.G. Hughes, ${ }^{1 *}$ R. Johns, ${ }^{2}$ B. Kiedrowski, ${ }^{1}$ R. Martz, ${ }^{1}$ S. Mashnik, ${ }^{1}$ \\ G. McKinney, ${ }^{2}$ D. Pelowitz, ${ }^{4}$ R. Prael, ${ }^{5}$ J. Sweezy, ${ }^{1}$ L. Waters, ${ }^{4}$ \\ T. Wilcox, ${ }^{2}$ and T. Zukaitis ${ }^{1}$ \\ ${ }^{1}$ Group XCP-3, Los Alamos National Laboratory \\ ${ }^{2}$ Group NEN-5, Los Alamos National Laboratory \\ ${ }^{3}$ Group XCP-7, Los Alamos National Laboratory, contractor \\ ${ }^{4}$ Group NEN-5, Los Alamos National Laboratory, contractor \\ ${ }^{5}$ Group XCP-3, Los Alamos National Laboratory, contractor \\ MS A143, Los Alamos NM 87545 USA \\ ${ }^{*}$ Corresponding Author, E-mail: hgh@lanl.gov
}

MCNP6 is simply and accurately described as the merger of MCNP5 and MCNPX capabilities, but it is much more than the sum of these two computer codes. MCNP6 is the result of six years of effort by the MCNP5 and MCNPX code development teams. These groups of people, residing in Los Alamos National Laboratory's X Computational Physics Division, Monte Carlo Codes Group (XCP-3) and Nuclear Engineering and Nonproliferation Division, Radiation Transport Modeling Team (NEN-5) respectively, have combined their code development efforts to produce the next evolution of MCNP. While maintenance and major bug fixes will continue for MCNP5 1.60 and MCNPX 2.7.0 for upcoming years, new code development capabilities only will be developed and released in MCNP6. In fact, the initial release of MCNP6 contains numerous new features not previously found in either code. These new features are summarized in this document. Packaged with MCNP6 is also the new production release of the ENDF/B-VII.1 nuclear data files usable by MCNP. The high quality of the overall merged code, usefulness of these new features, along with the desire in the user community to start using the merged code, have led us to make the first MCNP6 production release: MCNP6 version 1. High confidence in the MCNP6 code is based on its performance with the verification and validation test suites, comparisons to its predecessor codes, our automated nightly software debugger tests, the underlying high quality nuclear and atomic databases, and significant testing by many beta testers.

\section{KEYWORDS: Monte Carlo; particle transport; MCNP; MCNP6.}

\section{Introduction}

The particle radiation transport code MCNP, which stands for Monte Carlo N-Particle, is a general purpose three dimensional simulation tool that transports 37 different particle types for criticality, shielding, dosimetry, detector response, and many other applications.

Monte Carlo particle radiation transport methods have had an extensive history at Los Alamos National Laboratory (LANL) dating from the 1940s. Early creators of these methods include Drs. Stanislaw Ulam, John von Neumann, Robert Richtmyer, Nicholas Metropolis, and others, who investigated neutron transport issues on the first generation of computers. On March 11, 1947, John von Neumann sent a letter to Robert Richtmyer, leader of the Theoretical Division at Los Alamos, proposing the use of the statistical method to solve neutron diffusion and multiplication problems in fission devices. His letter was the first formulation of a Monte Carlo computation for an electronic computing machine. ${ }^{1}$ In 1947, while at Los Alamos, Fermi invented a mechanical device called FERMIAC11 to trace neutron movements through fissionable materials by the Monte Carlo Method. ${ }^{2}$ During the 1950s - 1960s, a number of special-purpose Monte Carlo codes were developed at LANL, including MCS, MCN, MCP, and MCG. These methods eventually found their way into a code called MCNG, which was first created in 1973 by merging a three dimensional neutron transport code $\mathrm{MCN}^{3}$, with the gamma transport code $\mathrm{MCG}^{4}$ In 1977 MCNG was merged with MCP, a Monte Carlo photon code with detailed physics treatment down to $1 \mathrm{keV}$, to more accurately model neutron-photon interactions. The resulting code, MCNP, originally stood for Monte Carlo Neutron Photon. In 1983, MCNP3 was released for public distribution to the Radiation Safety Information Computational Center (RSICC).

The meaning of MCNP changed to Monte Carlo N-Particle when electron transport, modeled after Sandia National Laboratory's Integrated TIGER Series (ITS), ${ }^{5}$ was added in 
1990. MCNP has been expanded ever since to include more and more particle types. In 1996, the LANL code LAHET was added to MCNP4B, creating a "Many-Particle MCNP Patch". 6,7 Although initially developed for the Accelerator Production of Tritium (APT) program, the utility of many-particle transport has found many applications and sponsors, and continued to grow as a separate code, MCNPX. ${ }^{8}$ In 2001-2002 MCNP4C was completely rewritten in modern Fortran 90, and was enhanced to support large scale parallelism using combined MPI message passing and OpenMP threading, resulting in MCNP5. In July 2006, a merger effort was started, beginning with MCNPX 2.6.B and adding it to a LANL version of MCNP5. During the development, new features independently developed for MCNPX and MCNP were also included in the merged code. The resulting code, MCNP6, incorporates a more complete set of transport physics and features than any previous member of the MCNP family of codes.

\section{Physics Models}

For users of traditional MCNP, the most striking new development will be MCNP6's ability to transport many more particle types at a greatly extended energy range. Specifically MCNP6 can transport neutrons and antineutrons, photons, electrons and positrons, positive and negative muons, protons and antiprotons, positive and negative pions, neutral lambdas and their antiparticles, positive and negative sigmas and their antiparticles, neutral and negative $\Xi$ and their antiparticles, positive and negative kaons, neutral kaons (as short- or long-lived kaons), negative and positive omegas, light ions (deuterons, tritons, ${ }^{3} \mathrm{He}$, and alphas), and heavy ions. (Electron and muon neutrinos and antineutrinos are also transported, but have no nuclear interactions. Neutral pions are transported without interactions, but may decay in flight.) The energy range that MCNP6 can contemplate has been extended to a domain of interest for a variety of high-energy applications, potentially reaching the $\mathrm{TeV}$ range or above. To transport this extended particle set at energies beyond the range of nuclear data tables, MCNP6 makes use of several different nuclear reaction models (sometimes called "event-generators"). The first model of intermediateand high-energy nuclear reactions, used initially in HETC and LAHET, ${ }^{10}$ was the Bertini INC, ${ }^{11}$ followed (by default, but not required) by the Multistage Preequilibrium Model (MPM $),{ }^{12}$ followed by the Dostrovsky et al. evaporation model $^{13}$ as implemented in the code EVAP by Dresner. ${ }^{14}$ If the compound nuclei produced after the INC and MPM stages of reactions are heavy enough to fission, the fission process is simulated either with the semi-phenomenological Atchison fission model, often referred in the literature at the Rutherford Appleton Laboratory (RAL) fission model, which is where Atchison developed it, ${ }^{15}$ or with the Fong statistical model of fission as implemented in the ORNL code HETFIS, ${ }^{16}$ often referred in the literature as the ORNL fission model. Bertini INC, MPM, EVAP, RAL, and HETFIS migrated from LAHET to MCNPX and later, to MCNP6. Bertini INC can be used (CEM3.03 is the default) in MCNP6 for reactions induced by nucleons and pions at energies up to $3.5 \mathrm{GeV}$ and it is always used to calculate such reactions on the light $\mathrm{d}, \mathrm{t},{ }^{3} \mathrm{He}$ and ${ }^{4} \mathrm{He}$ target-nuclei, independently of the model chosen in the MCNP6 input file. The second model, from historical point of view, migrated to MCNP6 via MCNPX from LAHET is the ISABEL INC. ${ }^{17}$ ISABEL is the default option in MCNP6 for reactions induced by $\mathrm{d}, \mathrm{t},{ }^{3} \mathrm{He},{ }^{4} \mathrm{He}$, and antinucleons at energies up to $1 \mathrm{GeV}$ per nucleon. If specified in the MCNP6 input file, ISABEL can be used to also simulate reactions induced by nucleons, pions, kaons, and heavy-ions at energies below 1 $\mathrm{GeV} /$ nucleon. Just like Bertini INC, ISABEL can be used with or without taking into account the preequilibrium reactions as described by MPM and it can describe the evaporation and fission reactions with EVAP, RAL, and HETFIS. A newer and recently improved model used by MCNP6 is the Cascade-Exciton Model (CEM) of nuclear reactions as implemented in the event-generator CEM03.03 ${ }^{18,19}$ CEM03.03 is used in MCNP6 as a default choice to calculate photonuclear reactions at energies up to $1.2 \mathrm{GeV}$, reactions induced by nucleons and pions at energies up to $3.5 \mathrm{GeV}$ reactions, and is the only model adopted by MCNP6 to simulate absorption of stopped muons. We recommend using it up to about $1 \mathrm{GeV}$ for light targets like $\mathrm{C}$ and up to about $5 \mathrm{GeV}$ for heavy targets like $\mathrm{U}$. CEM03.03 uses its own models to describe the preequilibrium, evaporation, and fission reactions. It considers also coalescence of nucleons into complex particles up to ${ }^{4} \mathrm{He}$ and Fermi break-up of excited or unstable nuclei with mass numbers up to $\mathrm{A}=12$ (see details in Refs. 18 and 19).

Another new and recently improved model used by MCNP6 is the Intra Nuclear Cascade model developed at Liege (INCL) by Joseph Cugnon in collaboration with colleagues from CEA Saclay, France and GSI, Germany. ${ }^{20}$ The version of INCL implemented so far in MCNP6 can describe successfully reactions induced by nucleons, pions, and complex particles $\mathrm{d}, \mathrm{t},{ }^{3} \mathrm{He}$, and ${ }^{4} \mathrm{He}$ at energies up to several $\mathrm{GeV}$. INCL always uses only the ABLA code developed at $\mathrm{GSI}^{21,22}$ to describe the evaporation and fission stages of reactions, independently of what MCNP6 users would chose for evaporation/fission models; INCL does not consider preequilibrium reactions. Newer and better versions of INCL and ABLA are planned for incorporating in a future version of MCNP6.

Finally, MCNP6 uses the Los Alamos version of the Quark-Gluon String Model (LAQGSM) as implemented in the event-generator LAQGSM03.03. ${ }^{19,23}$ LAQGSM03.03 is the default option to describe in MCNP6 reactions induced by heavy-ions, by photons at energies above $1.2 \mathrm{GeV}$, particle-nucleon interactions, as well as reactions induced by projectiles not considered by other models. LAQGSM was developed to describe reactions induced by almost all types of elementary particles and by nuclei at energies up to about $1 \mathrm{TeV} /$ nucleon. LAQGSM uses its own models to describe the preequilibrium, evaporation, and fission reactions; it considers also coalescence of nucleons into complex particles up to ${ }^{4} \mathrm{He}^{\prime}$ and Fermi break-up of excited or unstable nuclei with mass numbers up to $A=12$ (these are the same models used by CEM, but adjusted to LAQGSM; see details in Refs. 19 and 23). For lower energy neutrons, 
protons, photons, and electrons, evaluated nuclear data can also be used to sample nuclear and atomic interactions.

\section{Evaluated Nuclear Data}

The MCNP6 code represents one of a set of synergistic capabilities developed at Los Alamos that also includes the evaluated nuclear data files (ENDF), and the data processing code NJOY. The ENDF project is a collaboration among many US National Laboratories and universities, coordinated by the Cross Section Evaluation Working Group (CSEWG) and Brookhaven National Laboratory (BNL). LANL plays a leadership role in the evaluation and integral data testing CSEWG committees, and leads the theory, modeling, experiment, and evaluation work cross sections for actinides and light nuclei. The ENDF/B-VII.0 database was released in $2006,{ }^{24}$ and the latest version of the database (VII.1) was released in December 2011. ${ }^{25}$ The NJOY code developed by R.E. MacFarlane, and more recently Skip Kahler, takes the ENDF files and makes application data files for transport codes such as MCNP. It is used widely in the nuclear technology community for this purpose, as well as for computing certain quantities such as radiation heating and damage. ${ }^{26}$ MCNP has played a central role in the Validation and Verification (V\&V) of these ENDF data. ${ }^{27}$

The ENDF/B-VII.1-based data consists of 423 nuclides processed to seven temperatures suitable for reactor simulations. These files include secondary particle production data for a variety of particles. Depending on the nuclear data being used, neutron interaction data is available up to 20 , or more recently, $150 \mathrm{MeV}$. The newest thermal neutron scattering, $\mathrm{S}(\alpha, \beta)$, data (ENDF71SaB) is available for 21 different materials. Proton interaction data is available for 47 isotopes, up to energies of 120 or $150 \mathrm{MeV}$ are in the library endf70prot. Photoatomic data is available for photon and electron transport up to $100 \mathrm{GeV}$ and $1 \mathrm{GeV}$, respectively. Photonuclear data, including Nuclear Resonance Fluorescence (NRF), is available for 157 specific isotopes up to $150 \mathrm{MeV}$ in the endf7u library, but not all of these have been through extensive testing. The photonuclear data library for 13 isotopes in the LA150u library have been rigorously verified, however. Photonuclear reactions are not active in calculations by default. Above the respective energy for the above reactions, for all hadron interactions, and for all nuclides that have no data, interactions are based on theoretical models with empirical corrections. There are no nuclear interactions for leptons based on models, with the exception of negative muon at-rest nuclear capture, which uses data for X-ray emissions and CEM to simulate the effects of de-excitation on the nucleus. While MCNP6 will allow particles up to $100 \mathrm{TeV}$ in energy, only particle energies up to $1 \mathrm{TeV}$ have been reviewed for accuracy.

\section{Distribution and Availability}

In August 2011, forty-five advanced users were invited to beta test MCNP6_Beta1, providing valuable feedback relevant to a wide range of applications. In early 2012 and 2013, MCNP6 Beta 2 and Beta 3, respectively, source and executables were made available through RSICC (http://rsicc.ornl.gov/).
The production release was made available in May 2013. The MCNP6 distribution package is on three DVDs. The first DVD includes the MCNP5 1.60, MCNPX 2.7.0 and MCNP6 codes, with executables pre-built for Windows, Linux, and MacOSX platforms (pre-built MCNPX 2.7.0 executables for the MacOSX are not provided), as well as more than 1 gigabyte of PDF references. Most of these documents are from Los Alamos, but a few are from other DOE laboratories. The second and third DVD includes the nuclear and atomic data, including the ENDF/B-VII.1 release. Users should note that the ENDF/B-VII.1 data is stored in a variety of sub directories, unlike the previous releases. These sub directory paths are included in the xsdir, making all of these files accessible to (and backwards compatible with) MCNP5 and MCNPX. For FY11 and FY12, the DOE ASC program agreed to underwrite the distribution fees for MCNP6, making it free to the requestor. A similar agreement has been reached for subsidizing the production release in FY13.

For at least the next several years, MCNP6 will be distributed with MCNP5 1.60 and MCNPX 2.7.0. Users are encouraged to run their particular problems with MCNP6 and their previous code of choice. MCNP6 is a new code and is not expected to track (produce exactly the same random number and particle interaction sequence) either of its parent codes. Significant differences from either MCNP5 or MCNPX should have some explanation, such as the inclusion of new physics, different default behavior, new or improved data, or bug fixes. A document will be provided with MCNP6 listing known reasons for expected differences from MCNP5 or MCNPX. Any other differences should be discussed with the MCNP6 development team by sending an email to monp6@lanl.gov.

MCNP is one of the world's leading particle radiation transport codes, with an estimated user base of more than ten thousand users over its release history. In fact, in fiscal years 2009 and 2010, RSICC distributed 1514 and 1512 copies, respectively, of the MCNP5 and MCNPX package, to users in US government institutions, academia, and private companies worldwide. ${ }^{29}$ In the time between January, 2001 and October, 2011, there were 11,586 requests for MCNP, but it is not known how many of these requests are from the same user asking for a newer version of the code. ${ }^{29} \mathrm{MCNP}$ is RSICC's most widely requested and distributed computer code.

MCNP6 user support is provided in several different ways. The MCNP6 reference DVD contains several user manuals, hundreds of specific LANL MCNP publications, and discussions on Monte Carlo theory. A few other resources are provided, such as a database of MCNP input files of medical physics human phantoms. MCNP6 developers will provide limited feedback to the existing monp and monpx forums. The MCNP6 development team provides week long classes for MCNP beginners, and separate advanced classes in criticality, variance reduction, and sometimes other specific topics. Additional course content on the new MCNP6 features is also being developed, some of which has already been integrated into the classes. 


\section{MCNP6 New Features}

In addition to the code capabilities of MCNP5 and MCNPX, MCNP6 includes several significant new capabilities not found in either of the parent codes. These capabilities are described below.

\section{1) Adjoint-based sensitivity coefficients}

MCNP6 contains the new ability to compute sensitivity profiles ${ }^{31}$ of $k_{\text {eff }}$ with continuous-energy physics for cross sections, fission multiplicities $v$ and spectra $\chi$, and scattering distributions. This is useful for identifying the criticality benchmarks that are similar to applications being analyzed, which is important for understanding how well MCNP6 and its data libraries perform for that particular application through quantifying uncertainties and biases. This capability is utilized through the use of the ksen card.

\section{2) Geometry mesh file creation (Ref. 32)}

MCNP6 provides the initial file-based link to the LANL discrete ordinates neutron and photon code Partisn ${ }^{33}$, separately distributed by RSICC. The initial intent is to provide an easy way to perform Monte Carlo vs. SN comparisons, separating geometry, multigroup data, and methodology effects. The longer-range goal is to take the adjoint flux weights from Partisn to use within MCNP for weight windows. The initial capability takes MCNP's constructive solid geometry (CSG), creates a regular mesh of homogenized materials in 1D (r), 2D (rz, xy), or 3D (xyz), and writes a Partisn-style geometry file in the LNK3DNT file format, as well as reads a Partisn-style geometry for continuous energy neutron transport. This capability was first available in MCNP6 Beta 2 and is utilized through the mesh and dawwg cards.

\section{3) Unstructured mesh geometry}

MCNP6 now includes the capability to track and tally neutrons and photons on an unstructured mesh embedded as a mesh universe within MCNP's constructive solid geometry. The unstructured mesh geometry can be created with Abaqus/CAE. ${ }^{34}$ First and second order mesh element types with four, five, and six sides are supported; all six element types may exist simultaneously in a model. When geometry models are assembled from parts, gap and overlap regions may exist, giving rise to undefined and over-defined phase space; the unstructured mesh tracking algorithms successfully address these conditions. Results collected on the mesh are written to special output file that can be imported by Abaqus/CAE for visualization or thermo-mechanical analysis. Tracking through the unstructured mesh geometry can be competitive in run times with constructive solid geometries, but varies greatly with the geometry complexity. ${ }^{35}$ Reference 35 work shows that when performance from a mesh model with a moderate element count is compared to performance from a traditional model with few surfaces, the traditional model wins every time. However, our unpublished results indicate that this is not always the case when the models are put on equal footing; there are situations where tracking on the mesh is faster and when the geometry detail is significant, setup times for the mesh models are much shorter. Dose calculations of human phantoms using the unstructured mesh geometry are typically an order of magnitude slower in run time when compared to an identical voxel lattice geometry. Unstructured mesh memory usage is also typically several times larger than a comparable human phantom lattice geometry, but is lower for simplistic cases. ${ }^{36}$ Complex models are much quicker and easier to construct with a tool such as Abaqus/CAE plus the visualization of results is superior; to many users these capabilities are more important. When it comes to multi-physics integration, the unstructured mesh is the hands down winner in terms of meeting requirements. The unstructured mesh capabaility is fully compatible with MPI, shared memory threading, or hybrid methods utilizing both, parallelism. This capability was first available in MCNP6 Beta 2 and utilizes the embed and related cards. New in MCNP6 Beta 3 is the ability to do parallel setup and initialization of the unstructured mesh by decomposing individual Abaqus "parts" onto separate MPI processes. Also new in MCNP6 Beta 3 is that these unstructured mesh geometries can be plotted in the MCNP plotter.

\section{4) Low energy photon and electron transport for atomic cross sections}

MCNP6 has extended the minimum energy cutoff for photon transport down to $1 \mathrm{eV}$. The ENDF/B-VI.8 based photo-atomic cross sections are included in the photo-atomic library eprdata12, selected by requesting .12p photon transport tables on the ZAIDS or plib keyword. This new feature is not available by default. The default photon energy cutoff remains $1 \mathrm{keV}$, so the user must explicitly request a lower cutoff on the cut:p card, for example cut:p $j$ 1.0e-06 for the lowest possible cutoff. These lower energies can be specified on $s d e f$, de/df, and energy bin cards. While they are likely compatible with other energy-specific cards, not all possible combinations have been tested. This new feature is closely coupled with the following new capabilities in atomic relaxation. Users are cautioned that at very low energies, molecular and other effects become important for scattering and absorption, and these more complex effects are not yet included in the photon transport methods. In addition, electron transport has been extended down to 10 $\mathrm{eV}$. This feature was first available in MCNP6 Beta 2.

\section{5) Complete photon-induced atomic relaxation}

Complete element-specific relaxation processes, with the emission of fluorescent photons and Auger and Coster-Kronig electrons, can now be modeled in MCNP6. The necessary data (from ENDF/B-VI.8) are included in the interim library eprdata 12 , in $.12 \mathrm{p}$ photon tables. Going beyond previous MCNP treatments, which considered only K-shell and average L-shell transitions, the new treatment addresses the full detailed relaxation cascade, sampling all allowed transitions down to the photon and electron energy cutoffs, and providing a much more detailed prediction of the relaxation spectra. It should be noted that higher-order effects in which early transitions change the probabilities of later transitions in the same cascade are not modeled, and 
that relaxation from electron-induced atomic vacancies has not been implemented yet. This feature was first available in MCNP6 Beta 2.

\section{6) Explicit tracking of all charged particles in} magnetic fields (Ref. 37)

MCNP6 can model magnetic fields in two different ways. The card bfld can be used to model constant (dipole), square edge quadrupole, and quadrupole magnets with a fringe-field kick, magnetic fields that co-exist with low density materials, such as air. This new capability is an alternative to the existing COSY maps, ${ }^{38}$ which is a direct correlation of entry and exit particle states in a magnetic field, which can only be applied in a vacuum, i.e. a void cell. COSY maps are also specific to one particle type, and only one COSY map can exist at a spatial location, while the bfld capability correctly treats all charged particle types passing through a particular cell. This feature was first available in MCNP6 Beta 2.

\section{7) Nested dxtran spheres}

Multiple dxtran spheres, a form of variance reduction and population control, can now be nested inside of each other. More than one dxtran sphere may be nested inside a larger dxtran sphere and the nested dxtran spheres need not be concentric. The primary restriction is that the spherical surfaces must not intersect. This nesting allows dxtran particles to be directed to one or more regions of interest. This feature was first available in MCNP6 Beta 2.

\section{8) Uncollided secondaries}

An uncollided particle in MCNP was historically defined to be any particle that had not undergone a collision since its creation as a source particle or as a secondary particle. This definition, in which secondary particles are created as uncollided particles, makes separation of the contribution from the direct source and contribution from secondary particles difficult for the user. This is especially true when users employ track-length tallies in radiography applications instead of next-event estimators. A new cell card, unc, has been added to MCNP6 that allows the user to control if secondaries are born as uncollided or collided particles. This feature was first available in MCNP6 Beta 2.

\section{9) Time bins for mesh tallies}

Time bins have been added for the MCNP5-style mesh tallies. Users can specify time bin boundaries in units of shakes (1 shake is $10^{-8} \mathrm{sec}$ ) on the fmesh card. The tally results can be obtained as integrated over the time bin (units of $\mathrm{cm}^{-2}$ ), as an average rate (tally per unit shake), or as a tally per unit energy (tally per MeV per shake). Specific fmesh time bins can also be plotted in the mesh tally plotter. These time bins are added with the tint and tbin keywords on the fmesh card. Time bins were also added to the MCNPX-style mesh tallies.

\section{0) Enhanced photon form factors}

New form-factor data from ENDF/B-VI.8 for coherent and incoherent photon scattering are available in the interim photoatomic transport library eprdata12, which is selected by requesting . $12 \mathrm{p}$ photon transport tables. These data extend the treatment of coherent and incoherent scattering to higher energies and/or larger scattering angles. The interpolation algorithms for the form-factor data have also been corrected for validity over the enhanced energy/angle range. ${ }^{39}$ These improvements provide a more complete representation of photon scattering, and are especially important for backscattering of coherent photons. This feature was first available in MCNP6 Beta 2.

\section{1) Surface and cell flagging are now possible with MCNP5-style mesh tallies}

Mesh tallies can be used in combination with the surface $(s f)$ and cell $(c f)$ flagging tally cards. However, unlike the regular tallies, only one mesh tally, the flagged tally, is created. A separate mesh tally will need to be provided for unflagged tally results. This feature was first available in MCNP6 Beta 2.

\section{2) Upgrade to CEM03.03 and LAQGSM03.03}

The LANL high-energy physics models have now been updated to their most recent releases. The stand-alone version of CEM03.03 is available from RSICC, while the stand-alone version of LAQGSM03.03 is not. In comparison with the versions used by MCNPX 2.7.0, MCNP6 uses an upgrade of CEM03.03 allowing us to calculate much better fission cross section and fission fragment yields from ${ }^{181} \mathrm{Ta}$ and the nearby target-nuclei; several other unobserved bugs in CEM and LAQGSM were also fixed. These updates were included in MCNP6 Beta 2.

\section{3) Generation of gamma rays from muonic atoms}

When a negative muon survives to reach its lower energy cutoff, it attaches to an atom to form a negatively charged "muonic atom." The muon then relaxes by a series of transitions toward the most tightly bound shell, emitting fluorescent gamma rays, in analogy to the electronic transitions characterizing ordinary atomic relaxation. Both MCNPX and MCNP6 contain a programmed library for muonic gamma-production for a variety of isotopes of interest. A new feature of MCNP6 is the ability to read and sample from new data in independent data files. This capability is a work in progress, since an ACE-like format for such data files has not yet been developed. By default, this capability is on, and users should specify both photons and muons on the mode card. The current data files are available for 71 isotopes. This feature was first available in MCNP6 Beta 2.

\section{4) Pre-collision next event estimator (Ref. 40)}

A pre-collision next-event estimator for point detectors has been developed for MCNP6. This pre-collision estimator augments the post-collision next-event estimator that has historically been used for point flux estimation in MCNP. The pre-collision next-event estimator includes the contribution of all possible reactions before the collision isotope and resulting reaction are sampled. This procedure provides an improved expected estimate per collision, but 
with a significant increase in computational costs per collision. This improved sampling technique removes the requirement to suppress coherent scattering for photon transport problems that include photon next-event estimators The sampling of all possible scattering reactions generally provides an increase in the Figure of Merit (FOM) for most photon problems. This increase in the FOM can be significant when the contribution to a photon next-event estimator is primarily from forward coherent scattering. For most neutron problems there is typically not a large increase in the FOM. However, for both photons and neutrons the pre-collision next-event estimator increases the convergence rate as measured by the time to pass MCNP's 10 statistical checks. This option is enabled by the FTn command and the new keyword PDS (for point detector sampling) with a single value to select the desired pre-collision option. This feature was first available in MCNP6 Beta 2.

15) Double differential particle interaction cross section generator (Ref. 41)

The GENXS option on the tropt card in MCNP6 allows the application of high-energy nuclear interaction models in a cross section generation mode, without particle transport. A source may be specified inside a medium; each history will consist only of the interaction of the source particle at the source energy with the components of the medium. The tallied outcome from the event consists of the energies and direction cosines of the secondary particles and the recoil nuclei (in the laboratory system). Although one expects that, in normal applications, the material composition will be a single isotope, averaged results may be obtained for a natural multi-isotopic element or a complex composition. This feature was first available in MCNP6 Beta 2 .

\section{6) New MCNP6 Depletion Capabilities ${ }^{42}$}

MCNP6 depletion enables complete, relatively easy-to-use depletion calculations in a single Monte Carlo code. The enhancements described here help provide a powerful capability as well as dictate a path forward for future development to improve the usefulness of the technology. The MCNP6 depletion capability enhancements (on the BURN card) beyond MCNPX 2.7.0 include: (1) new performance enhancing parallel architecture that implements both shared and distributed memory constructs; (2) enhanced memory management that maximizes calculation fidelity; and (3) improved burnup physics for better nuclide prediction; (4) added swapb keyword on the burn card, which allows universe swapping at each time step during the burn to mimic fuel shuffling at the assembly and pin levels. These improvements, as well as their performance in the $\mathrm{H}$. B. Robinson fuel assembly benchmark, are detailed in the reference 42 .

\section{7) Cosmic Ray sources (SDEF card, PAR keyword) ${ }^{43}$}

A new cosmic-ray source option has been added to the SDEF card, PAR keyword. This PAR=cr,ch,ca option results in the production of cosmic protons and alphas, sampled from appropriate energy/angle distributions based on a calendar date specified on the DAT keyword, along with automatic normalization via the WGT keyword. Two interplanetary spectra are provided: (1) a recent development by BRI of UoD, and (2) a historic treatment presented by PRL of India. Both of these approaches can be applied to an Earth atmospheric application with the specification of the LOC keyword, in which case the geomagnetic modulation is treated.

\section{8) Support High-Fidelity Skymap Data Files}

The Cosmic Source feature requires the use of UoD skymap data files that include polar and azimuthal rigidity data on a terrestrial grid. Previously this grid was limited to 40 latitude and 40 longitude grid points. Release 4 of the UoD skymap files includes 59 latitude and 36 longitude grid points, so mods are needed to support this increased fidelity.

\section{9) 64 Bit Windows Plotting support}

The MCNP geometry and tally plotter now work with the 64 bit MCNP6 executable on Windows operating systems. The X11 dynamically linked library, created from the X11R6 source and compiled with a 64 bit Microsoft $\mathrm{C} / \mathrm{C}++$ compiler, X11_64.dll, is installed with the 64 bit windows MCNP6 executable.

\section{0) Background source option (SDEF card, PAR option)}

A database of background neutrons and gammas for different locations on the Earth's surface has been added and is available as a source particle type $(\mathrm{PAR}=\mathrm{BN}, \mathrm{BN}$, or $\mathrm{BG}$ for background neutrons, gammas or both respectively) on the SDEF card. This database is located in the file background.dat, which provides recent Release 2 spectra on a $10^{\circ} \times 10^{\circ}$ latitude/longitude grid. Reference 44.

\section{1) Pulse Height trigger tally option (FT card, PHL option)}

The pulse-height tally FT PHL option has been enhanced to allow for the specification of a trigger tally (TDEP keyword). The first contribution to the trigger tally sets the $\mathrm{T}=0$ reference time for the related pulse-height tally (i.e., T8 card). This capability enables one to overlay detector responses from different particle histories on a common time scale.

\section{2) Built-in photon detector response curves (FT card, PHL option)}

The pulse-height tally FT PHL option has been enhanced to allow for the specification of photon detector response curves (NaI-1, BGO-1, etc.). These response curves, which can be specified for each coincidence detector region, use Birk's law to convert charged-particle energy deposition to light output.

These obey the formula: $\mathrm{dL} / \mathrm{dE}=\mathrm{C} 0 /(1.0+\mathrm{C} 1 * \mathrm{~S})$ where $\mathrm{S}$ is the charged-particle stopping power at the particle track energy. The available functions are for the following detectors: ${ }^{3} \mathrm{He}, \mathrm{BF}_{3}$, Li glass, LiI, ZnS, NaI, BGO, CsI, BC400, HPGe. 
23) Capture trigger tally option (FT card, CAP keyword)

Similar to the FT PHL trigger option discussed above, the FT CAP feature has been enhanced to allow for the specification of a trigger tally (EDEP keyword). The first contribution to the trigger tally sets the $\mathrm{T}=0$ reference time for the related capture tally GATE entries. This capability enables one to overlay capture responses from different particle histories on a common time scale. The trigger tally specification should be flexible and enable a listing of one or more F6 tallies, along with an energy deposition threshold.

24) First-fission tally option (FT card, FFT keyword)

A new special tally treatment has been implemented for the FT card, called the First Fission Tally (FFT) option. This option segregates the associated F tally into FU bins based on the isotope of first fission. The related FU card contains a list of fissionable ZAIDs, and/or the MT values of 16 and 18 , and the score that is made to the associated F tally is placed in the FU bin based on which isotope corresponds to the first fission (or into the 0 bin if there was no fission or no other FU bin to score in, or into the 16 bin if the first reaction is [n,xn], or into the 18 bin if the first fission does not correspond to any isotopes listed on the FU card). This feature allows the user to see how a tally varies based on which reaction (currently $[\mathrm{n}, \mathrm{f}]$ or $[\mathrm{n}, \mathrm{xn}]$ ) occurs first in the particle history.The first fission tally separates tallies into bins according to which nuclide had the first induced fission. Identification of the first nuclide to undergo fission is useful for active interrogation methods to identify nuclear materials1. The average number of fission neutrons emitted by fission, $v$, differs from one nuclide to another. If a material of with unknown amounts of various fissionable nuclides is subject to a uniform source, the system multiplication will be proportional to the $v$ of the first nuclide undergoing fission2. Although not measured with an actual detector, this capability enables a cleaner analysis for detector design because the signal is subsequently proportional to the fission neutron production of the first fission nuclide.

\section{5) Delayed Beta treatment (ACT card)}

Enhancements were made to the delayed-particle treatment, including an improvement to the delayed-neutron spectra, the ability to read delayed-gamma data from the delay_library.dat file, and the inclusion of delayed-beta data and related algorithms. The particle type "e" is now allowed on the FISSION and NONFISS keywords of the ACT card. Reference 45.

26) Spontaneous beta source option (SDEF card, PAR keyword)

The spontaneous source option on the SDEF card was extended to include the production of neutrons and betas from spontaneous decay of unstable radionuclides. The unstable radionuclides can be specified in a material $(\mathrm{PAR}=\mathrm{sn}, \mathrm{sp}, \mathrm{sb})$ or as a particle type (PAR=ZAID); in either case one must specify the "\#" (heavy ion) particle type on the MODE card. With the specification of long-lived radionuclides, one should consider increasing the new stability half-life parameter (DBCN 10th entry), which increases the accuracy of the decay time-integration.

\section{7) Delayed-particles from photonuclear library interactions}

The library-based photonuclear collision routine was upgraded to include the ability to generate delayed particles from non-fission reactions (photofission already included this capability). However, the user should be warned that most photonuclear ACE libraries lump all secondary production into $\mathrm{MT}=5$, which prohibits the identification of a residual and thus delayed-particle production. ACE libraries that have been updated with specific reaction MT values can take advantage of this capability.

\section{8) New data path directories and default xsdir names}

To help organize the nuclear data files, many of the files are now stored within separate subdirectories. The highest level directory, pointed to by the DATAPATH environmental variable, contains the xsdir file (as it always has), but now contains the xdata and xmc directories. The xdata directory contains all of the nuclear data released from the nuclear data team, now located in XCP-5 (the Materials and Physical Data group). This includes the past ACE files for ENDF/B releases, including neutron, photoatomic, photonuclear and a few proton libraries. The xmc directory contains data libraries released from the MCNP6 development team, including more proton libraries and new photon, electron and atomic relaxation libraries that have been extended down to lower energies. Many additional files are still stored in the high level directory for backwards compatibility with MCNPX.

Furthermore, the naming convention for the xsdir filename has changed. The file xsdir_monp6.1 is the default searched for first by MCNP6. If this file is not found, then xsdir is searched for. Also note that libraries xsdir_mcnp6.1_endfb-7.0 and xsdir_mcnp6.1_endfb-7.1 were also created to allow users to easily select which set of nuclear data they wish to use by default (i.e. when no extension is listed on their ZAIDS, and no nlib keyword is used). A similar set has also been created for MCNP5. The MCNP6 version contains references to eprdata12 (which is incompatible with MCNP5 and MCNPX), while the MCNP5 version does not.

\section{9) New installers}

New windows command shell installers were developed to address difficulties with the unzipping program 7z.exe, which caused users on some Windows systems difficulties. The new windows installers do not use or include 7z.exe. Instead they use the unzip command in the Windows operating system, accessed through visual basic scripts. The installers on all systems have been re-written to address the multiple DVDs that make up the distribution and slight variations in the recommend installation paths. 


\section{0) Data library (only) driven transport for n,p,e} by default

During the merger process, we discovered that in some instances in MCNP6 it was possible for traditional simulations using neutron, photon and electron data table based transport (ie. MCNP5 simulations), to invoke nuclear interactions models. In these traditional criticality and shielding applications, at or below $100 \mathrm{MeV}$, models are poor predictors of near-threshold or resonance reactions, potentially leading to incorrect answers.

To protect these users from running input files with a one character typo on a ZAID and thus unexpectedly invoking model based transport, we made it a fatal error if any of the ZAIDs are not found in the xsdir. If the models are explicitly requested on an mphys card, or if any additional particles are requested on the mode card, then models will be used to calculate cross sections for isotopes not found in the xsdir.

\section{1) Continuous thermal scattering laws $S(\alpha, \beta)$}

MCNP6 may take full advantage of the new format thermal scattering $\mathrm{S}(\alpha, \beta)$ in ENDF/B-VII.1. The extension for these is $.20 \mathrm{t}$ (e.g., light-water is lwtr.20t). Earlier data scattered neutrons at discrete directions. The sampling probabilities were chosen to preserve integral quantities like $k_{\text {eff, }}$ but would result in "ray effects" for resolved or differential quantities like fluxes. The new data is continuous in energy and scattering angle. In principle, MCNP5 and MCNPX can read this data, but a bug in the sampling procedure present in both versions would lead to slightly incorrect answers and eventually would cause the code to crash or enter an infinite loop. Therefore, those wanting to use the ENDF/B-VII.1 continuous thermal scattering laws should exclusively use MCNP6.

\section{2) Perturbations for $\boldsymbol{k}_{\text {eff }}$}

The old perturbation capability, the differential operator or PERT, in MCNP5 and MCNPX may be used reliably in fixed-source or SDEF problems, but should only be used with great caution in eigenvalue or KCODE problems. The reason for this is that the methods in PERT do not consider the effect of the perturbation on the fission source. An integral method based on adjoint functions was developed in MCNP6 that correctly computes perturbations for $k_{\text {eff }}$ in KCODE problems. This may be invoked with the KPERT card.

\section{3) Updates to Delayed Particle Model Package}

Update delayed neutron so spectra are sampled from all DN emitters in a decay chain rather than just the spectrum of the precursor. Update delayed gammas so bin-wise spectra can be taken from the delay_library.dat file (instead of using only 25-bin data from the cinder.dat file). Update the delayed-particle package so delayed betas are produced, but only when the data is provided in the delay_library.dat file.

34) FMESH can now tally source points.

The FMESH tally can now tally (and therefore plot) the source point locations. This is accomplished by adding
TYPE=SOURCE on the FMESH card. This is expected to work with all other FMESH capabilities.

\section{5) FMESH can tally by the number of collisions}

The FMESH tally can now tally by the number of collisions a particle has had. This is accomplished by adding the INC keyword on the FMESH card. This is expected to work with all other FMESH capabilities.

\section{MCNP6 Development Process}

MCNP6 is considered risk level two, the second highest category of four levels. Risk level two software is treated as if failure of the software could result in temporary injury or illness to workers or the public. MCNP6 is not considered a risk level one, i.e. failure of the software would result in death, loss of life or limb, or permanent illness in the public or workers (i.e. one definition of risk level one software), because MCNP was not developed expressly for nuclear safety calculations, an explicit criterion for risk level one software.

The MCNP6 team uses TeamForge ${ }^{\circledR}$ software for source code and document version control and for tracking code defects and new features. TeamForge uses the concurrent version system (cvs) for version control. Each code developer has the ability to check-out the source code, make modifications, and check-in improvements. Each check-in must also reference a TeamForge artifact, which is a description of the background and intent of a particular new feature or defect fix. Artifacts query the developer regarding the status, category, priority, platform, and version of defects. Emails, input files, other documentation, and other artifacts can also be associated with each artifact. Artifacts also contain information about which developer owns the artifact and which developer is the artifact reviewer. Through this process, each line change in the source code has an associated artifact and developer. A web-based user interface allows developers to easily search by developer, artifact name or containing text, or subroutine. Changes in MCNP6 are required to be peer reviewed within the MCNP6 development team. Prior to check-in, each developer is required to ensure that his modifications pass the regression test suites on at least one computing platform with at least one compiler. Thirty minutes after the check-in, the automated continuous build and test system (CTBS) builds MCNP on several platforms with different compilers and runs several of the test suites, described below (Ref. 46). Differences from expected results in this automated system are monitored daily and developers who are responsible for differences are contacted.

The development history of each MCNP5 subroutine change has been maintained and updated this way since 2005 , when the team switched from different version control software. MCNP6 changes associated with the merger were associated with an overarching merger artifact, not individual features or defect corrections associated with the merger process. New features created in MCNP6 during the merger process, however, are identified by their own separate artifacts and check-ins. 


\section{Verification, Validation, and Test Suites}

The MCNP6 development team strives to deliver a high quality production code that works on many compilers and platforms. The confidence behind MCNP6 is supported by its favorable comparisons with benchmark experiments and, in some cases, analytical results. To a lesser degree, an extensive level of regression test processes and our code development process add to that confidence. Comparisons for the verification and validation $(\mathrm{V} \& \mathrm{~V})$ tests are automated, and html tables or plots of code results compared to experimental data or analytical results are created each week. These comparisons are available to the MCNP code developers and form the basis for the MCNP6 V\&V documents.

MCNP6 has 23 test directories containing several hundred specific problems testing that MCNP6 is running as expected. These tests span regression, verification and validation problems and are detailed by directory below. Each of these test suites has expected Linux and Windows Templates, for regression tests, or a set of analytical or benchmark values for $\mathrm{V} \& \mathrm{~V}$ tests. The MCNP6 team strives to make the calculated results of the test suites independent of computer platform, compiler and serial or parallel execution, where possible. For more information, see the "About the tests" or README file in many of the subdirectories in the MCNP6 distribution. Users are cautioned that regression test problems intentionally create fatal errors or otherwise exhibit poor geometry, source, tally or physics input practices. Regression tests are not to be imitated in production calculations.

\section{VII.A. DELAYED PARTICLES}

This validation test suite contains 6 test problems that simulate energy and time spectra from delayed neutron emissions from thermal neutron induced fission. These simulations are compared to microgram quantities of individual ${ }^{233} \mathrm{U},{ }^{235} \mathrm{U}$, and ${ }^{239} \mathrm{Pu}$ samples irradiated in a SLOWPOKE-2 research reactor for 60 seconds, and subsequently placed in a ${ }^{3} \mathrm{He}$ detector system and measured for 3 minutes ${ }^{47}$.

\section{VII.B. ELECTRONS}

This test suite contains 100 test problems that exercise various aspects of electron and coupled photon/electron transport. An important subset of these problems explore three different algorithms for the application of the Landau straggling theory for electron collisional energy loss. These are the "bin-centered" method, the ITS-style "nearest-group-boundary" method, and the more recently developed (and preferred) "energy and step specific" method. ${ }^{48}$ These tests show that the recommended algorithm (which is now the default in MCNP6) greatly reduces the occurrence of step-size-related artifacts in the energy-loss sampling for electrons. This test suite does not compare to experimental data.

\section{VII.C. KOBAYASHI}

The "Kobayashi Benchmarks" are added to the MCNP6 test suites. This set of $3 \mathrm{D}$ benchmark problems consists of simple geometries that contain at least one void region and one monoenergetic isotopic, cubic source region. ${ }^{49}$ Each configuration is simulated first with a purely absorbing and then with a fifty-percent scattering medium. Fluxes are calculated at various points throughout the geometries using point detector tallies. For the purely absorbing cases, there are exact solutions obtained using numerical integration. For the cases with scattering, reference solutions were computed by very long runs with the MVP Monte Carlo code. ${ }^{50}$ Overall, for two cases of each of the three problems, 136 different fluxes are compared between computed MCNP6 results and the reference.

\section{VII.D. LONG_INTEGER}

MCNP6 contains more than fifty integer variables that are always stored as 8-byte integers. This allows users to run more than 2.1 billion source particles on 32-bit operating system, for example. These six test problems help test that these large integer values can be read in the input parser, and that these variables do not overflow (i.e. become large negative integers) during execution of MCNP. Some of these tests may require hours or days to execute on some powerful LANL supercomputers.

\section{VII.E. MAG_FIELDS}

These 14 input tests exercise the magnetic field transport in air and down a proton radiography beam line. Effects on proton and electron transport from constant and quadruple magnetic fields are modeled.

\section{VII.F. MCNPX 65}

This is the set of eighty seven MCNPX regression test problems. Several of these test problems include continue runs, surface sources, ptrac file production, weight-window file creation and usage, and mixing nuclear data tables and models during neutron transport.

\section{VII.G. MCNPX EXTENDED}

This set of directories includes the MCNPX test problems from later MCNPX releases. They are divided into subdirectories based on the feature being exercised. See Table III for a more detailed listing of these inputs. In each of these directories, the file TheCount is a listing of the total number of output files created. Currently these tests are considered regression checks, but several of these problems exercise two different ways of doing the same thing. For example, the macrobody test problems mimic the same geometry as the conventional CGS.

\section{TABLE III}

Summary of MCNPX_Extended Test Problems.

\begin{tabular}{|l|l|l|}
\hline Directory & $\begin{array}{l}\text { Number } \\
\text { of test } \\
\text { problems }\end{array}$ & Notes \\
\hline avr & 22 & Advanced variance reduction \\
\hline class & 25 & $\begin{array}{l}\text { Simple problems used in the } \\
\text { week-long MCNP classes }\end{array}$ \\
\hline
\end{tabular}




\begin{tabular}{|c|c|c|}
\hline classgeom & 20 & $\begin{array}{l}\text { Examples of more advanced } \\
\text { geometries, including macrobody } \\
\text { hexagonal prisms, cell based } \\
\text { rejection, filling universes }\end{array}$ \\
\hline classvar & 10 & $\begin{array}{l}\text { Class problems on variance } \\
\text { reduction methods }\end{array}$ \\
\hline heavyions & 9 & $\begin{array}{l}\text { Test problems focusing on heavy } \\
\text { ion transport }\end{array}$ \\
\hline mbody & 19 & $\begin{array}{l}\text { Test reading and transporting } \\
\text { particles through all the } \\
\text { macrobodies }\end{array}$ \\
\hline phys & 28 & $\begin{array}{l}\text { Tests a variety of coupled particle } \\
\text { physics interactions, data } \\
\text { sampling routines, }\end{array}$ \\
\hline push & 24 & Variety of simple historic tests \\
\hline test $27 \mathrm{a}$ & 12 & $\begin{array}{l}\text { Tests the new features released in } \\
\text { MCNPX 2.7.A: tally tagging, } \\
\text { activation, embedded sources }\end{array}$ \\
\hline test $27 b$ & 13 & $\begin{array}{l}\text { Tests the new feature released in } \\
\text { MCNPX 2.7.B: spontaneous } \\
\text { fission weighting, charged ion } \\
\text { creation from nuclear reactions, } \\
\text { LET tallies and dose equivalent } \\
\text { tallies, }\end{array}$ \\
\hline test $27 \mathrm{c}$ & 10 & $\begin{array}{l}\text { Tests the new feature released in } \\
\text { MCNPX 2.7.C: muons, new ptrac } \\
\text { features, time dependant mesh } \\
\text { tallies, delayed neutron treatments }\end{array}$ \\
\hline test $27 \mathrm{~d}$ & 19 & $\begin{array}{l}\text { This is a test suite to test the new } \\
\text { feature released in MCNPX 2.7.D: } \\
\text { improved form factors, cyclic } \\
\text { time bins, elemental residuals, } \\
\text { coincidence, signal to noise PDFs. }\end{array}$ \\
\hline test27e & 21 & $\begin{array}{l}\text { Tests the new feature released in } \\
\text { MCNPX 2.7.E: fission } \\
\text { multiplicity, } \\
\text { background sources, nested file } \\
\text { read statements, }\end{array}$ \\
\hline testburn & 16 & $\begin{array}{l}\text { Tests that exercise the } \\
\text { production/depeletion features } \\
\text { relating to the burn card. }\end{array}$ \\
\hline testdndg & 18 & $\begin{array}{l}\text { Tests relating to delayed neutron } \\
\text { and gamma emission spectra }\end{array}$ \\
\hline testincl & 42 & $\begin{array}{l}\text { Tests relating to high energy } \\
\text { particle transport, energy } \\
\text { deposition, photonuclear physics }\end{array}$ \\
\hline testmcnp & 31 & $\begin{array}{l}\text { The set of regression test } \\
\text { problems from MCNP4B }\end{array}$ \\
\hline testmesh & 10 & $\begin{array}{l}\text { Tests mesh tallies with various } \\
\text { geometries and particles. }\end{array}$ \\
\hline testmix & 13 & $\begin{array}{l}\text { Tests of the transition between } \\
\text { using models and data tables for } \\
\text { transport. }\end{array}$ \\
\hline testpht & 58 & $\begin{array}{l}\text { Tests of the Pulse Height Tally } \\
\text { with variance reduction }\end{array}$ \\
\hline testxnew & 19 & $\begin{array}{l}\text { Test of previous version of } \\
\text { MCNPX }\end{array}$ \\
\hline
\end{tabular}

\begin{tabular}{|l|l|l|}
\hline testxold & 35 & $\begin{array}{l}\text { Test of even older version of } \\
\text { MCNPX }\end{array}$ \\
\hline zrecoil & 37 & Test of light ion recoil \\
\hline
\end{tabular}

\section{VII.H. MUONS}

These 22 test problems exercise the creation of characteristic muonic X-rays using older and newer methods. This suite is mostly used by the developers to probe the effects of changing particular muon physics options. There is no comparison to experimental data.

\section{VII.I. PERFORMANCE}

These four tests are used to evaluate runtime performance for a variety of features. They are intended to be run repeatedly to collect an average execution time. The input files include cases representing criticality, pulse height tally variance reduction, photon transport through a lattice representation of a human head, and a generic porosity tool used in nuclear oil well logging.

\section{VII.J. PHOTONS}

This test suite of fifty five tests was used by the developers as they extended photon transport down to $1 \mathrm{eV}$ to investigate certain physics effects.

\section{VII.K. PHTVR}

This set of 209 problems test the pulse height tallies with several combinations of variance-reduction methods.

\section{VII.L. REGRESSION}

The standard MCNP6 Regression Test Suite comprises one hundred and fourty eight problems, with new tests added to cover code features or to explicitly confirm that particular bugs are fixed. The regression tests do not verify code correctness; they are used only for the purpose of detecting unintended changes to the code and for installation testing. This directory also contains sixteen regression tests of transport through unstructured meshes.

\section{VII.M. VALIDATION_CEM}

These benchmarks exercise the ability to create and transport high energy particles using CEM. ${ }^{51,52}$ Tests, and their experimental comparisons to data, are of the following categories: isotope production by bremsstrahlug photons of energies from $30 \mathrm{MeV}$ to $4.5 \mathrm{GeV}$ impacting a thin ${ }^{93} \mathrm{Nb}$ target and double-differential spectra of protons spectra at several angles produced by bremsstrahlug photons of energies from $30 \mathrm{MeV}$ to $4.5 \mathrm{GeV}$ on a thin ${ }^{12} \mathrm{C}$ target; delayed neutron emitters production from a thin ${ }^{238} \mathrm{U}$ target bombarded with $1 \mathrm{GeV}$ protons; neutron and gamma spectra resulting from $18 \mathrm{MeV}$ protons on water containing ${ }^{18} \mathrm{O}$; backward and off-axis angle spectra of neutrons resulting from $1200 \mathrm{MeV}$ protons on thick Fe cylinders; proton and complex particles spectra (gas production) from thin ${ }^{238} \mathrm{U}$, ${ }^{197} \mathrm{Au}$, and ${ }^{209} \mathrm{Bi}$ thin targets bombarded with nucleons of energies below $1 \mathrm{GeV}$, to name just a few. 


\section{VII.N. VALIDATION_CRITICALITY}

The criticality validation suite ${ }^{53}$ consists of thirty-one problems from the International Handbook of Evaluated Criticality Benchmark Experiments. ${ }^{54}$ It contains cases for a variety of fuels, including ${ }^{233} \mathrm{U}$, highly enriched uranium (HEU), intermediate-enriched uranium (IEU), low-enriched uranium (LEU), and plutonium in configurations that produce fast, intermediate, and thermal spectra. For each type of fuel, there are cases with a selection of moderators, reflectors, spectra, and geometries. The cases in the suite were chosen to include a broad range of configurations. The suite was modified to permit running with either ENDF/B-VI data libraries or the newer ENDF/B-VII.0 data libraries.

\section{VII.O.VALIDATION_CRIT_EXPANDED}

This directory contains one hundred nineteen problems from the International Handbook of Evaluated Criticality Benchmark Experiments and is an extension of the VALIDATION_CRITICALITY suite. ${ }^{55,56}$

\section{VII.P. VALIDATION_LAQGSM}

It is similar to the VALIDATION_CEM suite, but intended to test the high-energy event generator LAQGSM03.03.$^{51,57}$ LAQGSM is the only model used by MCNP6 allowing simulated interactions with heavy-ions and with almost all types on elementary particles at energies up to about $1 \mathrm{TeV} /$ nucleon. This directory contains eighteen comparisons of resulting neutrons, protons, deuterons, tritons, ${ }^{3} \mathrm{He},{ }^{4} \mathrm{He}$, pion, and kaon double-differential spectra from various reactions induced by protons, photons, and heavy-ions with incident energies up to $400 \mathrm{GeV}$. Additionally, several comparisons are made of the production of radioisotope fragments from thin gold, silver, copper, and silicon targets bombarded with protons and heavy-ions with energies up to $800 \mathrm{GeV}$. Neutron spectra from a thin carbon target bombarded with a 290 $\mathrm{MeV} /$ nucleon ${ }^{12} \mathrm{C}$ beam, of interest to cancer treatment with a carbon-beam, are also investigated.

\section{VII.Q.VALIDATION_ROSSI_ALPHA}

MCNP6 has the capability to compute the point-kinetics parameter Rossi-Alpha for a system at delayed critical. For such a system, Rossi-alpha is minus the ratio of the effective delayed neutron fraction to the effective neutron generation time. Here the term "effective" implies that the quantities are importance or adjoint weighted. The Rossi-Alpha validation suite includes thirteen criticality benchmarks and assesses how well MCNP6 and associated nuclear data (e.g., ENDF/B-VII.0) can match experimentally measured values.

\section{VII.R. VALIDATION_SHIELDING}

The radiation shie-ding validation suite contains thirty seven inputs in three subcategories: time-of-flight neutron spectra from pulsed spheres, ${ }^{55}$ neutron and photon spectra at shield walls within a simulated fusion reactor, and photon dose rates from shielded sources and skyshine. Two of the cases are coupled neutron-photon calculations, while the others are exclusively neutron or photon calculations. This suite was overhauled to generate plots comparing calculated results against experimental data.

\section{VII.S. VERIFICATION_KEFF}

Reference provides a set of seventy-five criticality problems found in the literature for which exact analytical solutions are known. ${ }^{60}$ Number densities, geometry, and cross section data are specified exactly for these problems. As a part of MCNP6 verification, ten of these analytical benchmark problems are run to high precision.

\section{VII.T. VERIFICATION_SHIELDING_SVDM ${ }^{61}$}

Seventy eight shielding benchmark inputs were provided by Steven van der Marck (SVDM), in the categories of fusion neutronics time of flight, LLNL pulsed spheres, NIST water spheres, and Oktavian 14-MeV neutron source. Measured benchmark values were not provided with these inputs.

A small addition to the overall confidence of MCNP6 is its use of automated regression testing suites. MCNP6's $\sim 900$ problem test suites are run nightly on Windows 64, Linux 32, and Linux 64 bit computers, with Intel 12, Portland, and GFortran compilers; but, each compiler is not tested on each operating system. By compiling with this variety of compilers, the development team is more likely to find and correct coding defects. To exercise parallel capability, the regression suite is run nightly in serial, openmpi, omp, and openmpi+omp modes with the Intel 12 compiler on Linux 64. The serial tests are also compiled with array overflow and use of uninitialized variable debugging checks. When the Intel test coverage tool is applied to parallel execution of the ELECTRONS, FEATURES, MAG_FIELDS, MCNPX_65, MCNPX_EXTENDED, MUONS PHOTONS, PHTVR, POINT_KINETICS, and REGRESSION suites, we found that $91 \%$ of the files are tested, as are $79 \%$ of the functions, and $65 \%$ of the blocks. In this context, a block is defined as portion of code for which the instructions are executed exactly once, in order, with one entry and exit point. A large fraction of the remaining files and functions are associated with plotting. The results of these tests (more than 20,000 tests) are displayed on a large video screen in the XCP-3 lounge area. Our experience is that this highly visible status report results in faster identification of code compile failures and unintended impacts on regression suites, and therefore faster resolution of these issues. This test system was created by Charles Zeeb in the scripting language Perl, and is tightly integrated with the SQL database PostgreSQL. Additional parallel and compiler tests are performed on versions prior to a release.

\section{VII.U. FEATURES}

Recognizing that regression tests should be more appropriately ordered by what features they test, this directory is broken into regression tests for five new features. DAWWG tests the ability for MCNP6 to create a mesh of constructive solid geometries and write the appropriate 
LNK3DNT (structured mesh) files. The FMESH_INC directory contains five tests of the interaction of the fmesh card and the unc card. The Model_Dev directory contains 40 tests of the high-energy $(100 \mathrm{MeV}-\mathrm{TeV})$ particle interactions. Unlike the Validation test suites for CEM and LAQGSM below, these tests are not intended to be compared to experimental results. The Point_Detectors test suite contains 29 tests of photon transport and resulting tallies by point detectors. The UNC directory contains eleven tests for the collided or uncollided secondary particle production features.

\section{Future MCNP6 Development}

MCNP6 continues development in several widely disparate fields of Monte Carlo particle transport. We continue to improve its physics, either by improving our own transport algorithms or incorporating those developed by others. We are currently interested in adding molecular interaction cross sections for both photons and electrons, and improving the optical light transport with reflection and refraction. Improving the energy and time signatures of delayed particle emissions also continues to be a focus area. Most of the interaction physics today in MCNP assumes that average quantities (number and energies of products) are correct. While necessary a decade ago to fit within computer hardware requirements, this limitation is no longer necessary and many users are now interested in correlations of emitted neutrons and gammas.

MCNP6 will continue to merge the unstructured mesh capability with the other +features. Improvements to the temperature effects on neutron interactions are under development. Specific MCNP6 development already underway, and potential candidates for the next production release:

1) Data Library based light ion transport. The source particles (nuclei of ${ }^{1} \mathrm{H},{ }^{2} \mathrm{H},{ }^{3} \mathrm{H},{ }^{3} \mathrm{He},{ }^{4} \mathrm{He},{ }^{6} \mathrm{Li}$ and ${ }^{7} \mathrm{Li}$ ) will be able to use the recently created ACE files for particle transport less than $150 \mathrm{MeV}$. This is expected to drastically improve accuracy of low-energy near threshold reactions, which previously used theoretical models.

2) Heavy charge particle transport in the Unstructured Mesh.

3) Use of weight windows variance reduction in the Unstructured Mesh.

4) Use of FMESH tally mesh to calculate surface tally results.

5) On-The-Fly Neutron Doppler Broadening.

6) Update to the newest version of INCL.

7) Compton image tally options

8) Correlated prompt secondary particle production via CGM

9) Correlated delayed particle production

10) Updated release of the background.dat file

11) Spontansous neutron and beta source options

12) Improved delayed-particle time integration for long-lived radioisotopes

13) Delayed-particle energy biasing

14) Further improvements in electron-photon transport
We will also attempt to anticipate what the next generation of supercomputers will look like, and how to exploit their massive number of processors, and possibly even their hardware acceleration. We are in the process of investigating Fortran 2003 features, specifically the co-array capabilities, for advanced parallelism with lower communication overhead.

It is the intent of the MCNP developers to produce publically available MCNP6 updates every six months to one year. Although the MCNP source and executables are only available through RSICC, source code patches may be posted to the MCNP6 website, mcnp.lanl.gov. Users who have the ability to compile (which is not limited by cost since GFortran and gcc are free) and who possess the source from RSICC can produce their own executables; other users will need to request a new executable from RSICC.

\section{Citations}

We encourage authors who use and/or publish results from MCNP 5/X/6 simulations to use the following citations, as well as cite the full version number of the code used. This allows the development team to have a more accurate understanding of the wide variety of applications that MCNP is being used for, as well as total usage. Please note that MCNP6 journal article and the MCNP5 Overview and Theory Manual are available to anyone, while the MCNP6 and MCNPX manuals are export controlled.

\section{MCNP6:}

T. Goorley, M. James, T. Booth, F. Brown, J. Bull, L.J. Cox, J. Durkee, J. Elson, M. Fensin, R.A. Forster, J. Hendricks, H.G. Hughes, R. Johns, B. Kiedrowski, R. Martz, S. Mashnik, G. McKinney, D. Pelowitz, R. Prael, J. Sweezy, L. Waters, T. Wilcox, and T. Zukaitis, "Initial MCNP 6 Release Overview", LA-UR-11-07082, Los Alamos National Laboratory, also Nuclear Technology, 180, pg 298-315 (Dec 2012).

\section{MCNP5:}

X-5 MONTE CARLO TEAM, "MCNP - A General Monte Carlo N-Particle Transport Code, Version 5, Volume I: Overview and Theory," LA-UR-03-1987, Los Alamos National Laboratory (April 2003).

\section{MCNPX:}

"MCNPX User's Manual Version 2.7.0," LA-CP-11-00438, D. B. PELOWITZ, Ed., Los Alamos National Laboratory (Apr. 2011).

Users are also encouraged to cite the most recent nuclear data reference:

ENDF/B-VII.1 nuclear data:

M.B. Chadwick, M. Herman, P. Oblo`zinsk'y, M.E. Dunn, Y. Danon, A.C. Kahler, D.L. Smith, B. Pritychenko, G. Arbanas, R. Arcilla, R. Brewer, D.A. Brown, R. Capote, A.D. Carlson, Y.S. Cho, H. Derrien, K. Guber, G.M. Hale, S. Hoblit, S. Holloway, T.D. Johnson, T. Kawano, B.C. Kiedrowski, H. Kim, S. Kunieda, N.M. Larson, L. Leal, J.P. 
Lestone, R.C. Little, E.A. McCutchan, R.E. MacFarlane, M. MacInnes, C.M. Mattoon, R.D. McKnight, S.F. Mughabghab, G.P.A. Nobre, G. Palmiotti, A. Palumbo, M.T. Pigni, V.G. Pronyaev, R.O. Sayer, A.A. Sonzogni, N.C. Summers, P. Talou, I.J. Thompson, A. Trkov, R.L. Vogt, S.C. van der Marck, A. Wallner, M.C. White, D. Wiarda, P.G. Young, "ENDF/B-VII.1 Nuclear Data for Science and Technology: Cross Sections, Covariances, Fission Product Yields and Decay Data," LA-UR 11-05121, Los Alamos National Laboratory, also: Nuclear Data Sheets, 112 Issue 12, pg. 2887-2996 (Dec 2011)

\section{Conclusion}

MCNP6 is now available from RSICC and we encourage MCNP5 and MCNPX users to migrate to this code. MCNP6 will not exactly recreate the particle tracks of either MCNP5 nor MCNPX because several default options have been changed. Users should be aware that this first production release does contain several limitations. Although individual MCNP5 and MCNPX features work, full inter-operability between MCNP5, X and 6 features does not necessarily exist, and this large combination of features has not been extensively tested. While there has been a significant amount of $\mathrm{V} \& \mathrm{~V}$ behind MCNP6 already, and this $\mathrm{V} \& \mathrm{~V}$ is made available with the MCNP6 distribution, users have been cautioned and continue to be cautioned that they are responsible for $\mathrm{V} \& \mathrm{~V}$ for their own particular application of the code. It is expected that users of this initial release apply MCNP6 to the problems that they know well and have some intuition about, and report back to the MCNP6 team (email mcnp6@lanl.gov) their findings, either pro or con. Evidence for appropriate performance of MCNP6 should be added to the MCNP6 documentation and verification test suites, and evidence of discrepancies, especially discrepancies with MCNP5 1.60 or MCNPX 2.7.0, should be submitted for investigation. Users are also encouraged to read the accompanying MCNP5/X-to-MCNP6 Migration Notes and MCNP6 Known Issues document.

\section{Acknowledgements}

MCNP development over the last ten years has been supported by funding from the US Department of Energy's National Nuclear Security Administration-Advanced Simulation and Computing (NNSA-ASC), Nuclear Criticality Safety Program (NCSP), Department of Defense, and Department of Homeland Security. We thank these sponsors.

The MCNP6 code developers thank the authors of the other codes, modules and libraries who have allowed their algorithms to be incorporated into and distributed with MCNP6. Although typically developed at other DOE national laboratories, several algorithms were developed at other international institutes. The high-energy (typically $100 \mathrm{~s}$ of $\mathrm{MeV}$ to $10 \mathrm{~s}$ of $\mathrm{GeV}$ or higher) nuclear reaction models (event-generators) CEM and LAQGSM were initially proposed at the Joint Institute for Nuclear Research (JINR), Dubna, Russia, and developed, improved, and maintained thereafter at LANL; INCL + ABLA was developed initially at the Liege University, Belgium, and GSI, Darmstadt, Germany, with important contributions from researchers of CEA/Saclay, France. Lower energy physics contributions include the Sandia National Lab code ITS 3.0 for electron transport and LLNL's fission multiplicity model for both photon and neutron induced fissions. The LANL CINDER team and other T Division contributors are responsible for the radioactive isotope cross sections (for production and depletion) as well as the decay emission data. We also would like to thank the RSICC for their many years of distributing MCNP.

Finally, we also thank our users, especially those users who provide us with insight into what is not working as expected, what features would be more useful, and the code's performance against experiments. Those users who take the time to create small test problems and fully describe suspected code defects are extremely valuable to us, and they will be acknowledged with individual certificates and listed on the MCNP6 website, mcnp.lanl.gov. Many other users make the effort to provide detailed feedback to those who post questions to the monp email forums.

Those users who have provided extensive feedback and we especially wish to thank by name are: Joshua Bergman (ARA), Jamie Cash (SNL), Lila Chase (LLNL), John DeMarco (UCLA), Paul Goldhagen (DHS), Allen Harvey (TASC), Hans Kruger (LLNL), Chuck Lebeda (LANL), Ronald McConn Jr (PNNL), Brad Micklich (ANL), Dudley A. Raine, III, Robert Singleterry (NASA), Madison Sellers (RMC), Steven van der Marck (NRG)

\section{References}

1. N. C. COOPER, Ed., From Cardinals to Chaos - Reflections on the Life and Legacy of Stanislaw Ulam, Cambridge University Press, New York (1989).

2. N. METROPOLIS and S. ULAM, "The Monte Carlo Method," J. American Statistical Association, 44, 335 (1949).

3. E. D. CASHWELL et al., "MCN: A Neutron Monte Carlo Code," LA-4751, Los Alamos Scientific Laboratory (1972).

4. E. D. CASHWELL et al., "Monte Carlo Photon Codes: MCG and MCP," LA-5157-MS, Los Alamos Scientific Laboratory (1973).

5. J. A. HALBLEIB et al., "ITS Version 3.0: The Integrated TIGER Series of Coupled Electron/Photon Monte Carlo Transport Codes," SAND91-1634, Sandia National Laboratory (1992).

6. H. G. HUGHES and L. S. WATERS "Many-Particle MCNP," Memo, XTM:96-91, Los Alamos National Laboratory (1996).

7. H. G. HUGHES, R. E. PRAEL, and R. C. LITTLE, "MCNPX The LAHET/MCNP Code Merger," Memo XTM-RN 97-012, Los Alamos National Laboratory (1997).

8. H. G. HUGHES et al., "MCNPX ${ }^{\mathrm{TM}}$ - The LAHET ${ }^{\mathrm{TM}} / \mathrm{MCNP}^{\mathrm{TM}}$ Code Merger," LA-UR-97-1638, Los Alamos National Laboratory (1997), presented at SARE-3, Simulation of Accelerator Radiation Environments, Tsukuba, Japan, May 7-9, 1997.

9. X-5 MONTE CARLO TEAM, "MCNP - A General Monte Carlo N-Particle Transport Code, Version 5 - Volume I: Overview and Theory," LA-UR-03-1987, Los Alamos National Laboratory (2003).

10. R. E. PRAEL and H. LICHTENSTEIN, "User Guide to LCS: The LAHET Code System," LA-UR-89-3014, Los Alamos National Laboratory (1989).

11. H. W. BERTINI, "Low-Energy Intranuclear Cascade Calculation," Phys. Rev., 131, 1801 (1963); H. W. BERTINI, "Intranuclear Cascade Calculation of the Secondary Nucleon 
Spectra from Nucleon-Nucleus Interactions in the Energy Range 340 to $2900 \mathrm{MeV}$ and Comparison with Experiment," Phys. Rev., 188, 1711 (1969).

12. R. E. PRAEL and M. BOZOIAN, "Adaption of the Multistage Preequilibrium Model for the Monte Carlo Method," LA-UR-88-3238, Los Alamos National Laboratory (1988).

13. I. DOSTROVSKY, Z. FRANKEL, and G. FRIEDLANDER, "Monte Carlo Calculations of Nuclear Evaporation Processes. III. Application to Low-Energy Reactions," Phys. Rev., 116, 683 (1959).

14. L. DRESNER, "EVAP - A Fortran Program for Calculating the Evaporation of Various Particles from Excited Compound Nuclei," ORNL-TM-196, Oak Ridge National Laboratory (1962).

15. F. ATCHISON, "Spallation and Fission in Heavy Metal Nuclei under Medium Energy Proton Bombardment," in: Targets for Neutron Beam Spallation Sources, Jul-Conf-34, Kernforschungsanlage Julich GmbH (1980).

16. J. BARISH et al., "HETFIS High-Energy Nucleon Meson Transport Code with Fission," ORNL/TM-7882, Oak Ridge National Laboratory (1981);

F. S. ALSMILLER et al., "A Phenomenological Model for Particle Production from the Collisions of Nucleons and Pions with Fissile Elements at Medium

Energies," Nucl. Sci. Eng., 79, 147 (1981)

17. Y. YARIV and Z. FRANKEL, "Intranuclear Cascade Calculation of High-Energy Heavy-Ion Interactions," Phys. Rev. C, 20, 2227 (1979)

18. S. G. MASHNIK and A.J. SIERK, "CEM03.03 User Manual," LA-UR-12-01364, Los Alamos National Laboratory (2012).

19. S. G. MASHNIK et al., "CEM03.03 and LAQGSM03.03 Event Generators for the MCNP6, MCNPX, and MARS15 Transport Codes," LA-UR-08-2931, Los Alamos National Laboratory (2008), invited lectures presented at the Joint ICTP-IAEA Advanced Workshop on Model Codes for Spallation Reactions, ICTP, Trieste, Italy, February 4-8, 2008, Los Alamos (2008).

20. A. BOUDARD et al., "Intranuclear cascade model for a comprehensive description of spallation reaction data," Phys. Rev. $C, 66044615$ (2002).

21. J.-J. GAIMARD and K.-H. SCHMIDT, "A Reexamination of the Abrasion-Ablation Model for the Description of the Nuclear Fragmentation Reactions," Nuc. Phys. A, 531, 709 (1991).

22. A. R. JUNGHANS et al., "Projectile-Fragment Yields as a Probe for the Collective Enhancements in the Nucelear Level Density," Nuc. Phys. A, 629, 635 (1998).

23. K. K. GUDIMA, S. G. MASHNIK, and A. J. SIERK, "User Manaul for the code LAQGSM," LA-UR-01-6804, Los Alamos National Laboratory (2005).

24. M. B. CHADWICK et al., "ENDF/B-VII.0: Next generation evaluated nuclear data library for nuclear science and technology," Nuclear Data Sheets, 107, 2931 (2006).

25. M. B. CHADWICK et al., "ENDF/B-VII.1: Nuclear Data for Science and Technology: Cross Sections, Covariances, Fission Product Yields and Decay Data," Nuclear Data Sheets, 112, 2887 (2011).

26. R. E. MACFARLANE and A. C. KAHLER, "Methods for Processing ENDF/B-VII with NJOY," Nuclear Data Sheets, 111, 2739 (2010)

27. S. C. van der MARCK, "Benchmarking ENDF/B-VII.0," Nuclear Data Sheets, 107, 3061 (2006).

28. K. ALVIN, N. FRAZIER and R. MEISNER, "Advanced Simulation and Computing National Code Strategy," NA-ASC-108R-09-Vol.1-Rev.0,

US National Nuclear Security Administration, http://nnsa.energy.gov/sites/default/files/nnsa/inlinefiles/ASC-Code -Strategy.pdf (2009).
29. Private communication from Dr. Bernadette Kirk, Director, Radition Safety Information Computational Center, Oak Ridge, TN, November 2011

30. M. JAMES, "MCNPX to MCNP6 Migration Notes," LA-UR-11-01832, Los Alamos National Laboratory (2011).

31. B. C. KIEDROWSKI AND F. B. BROWN, "Adjoint-Based k-Eigenvalue Sensitivity Coefficients to Nuclear Data Using Continuous-Energy Monte Carlo," Nucl. Sci. Eng., 174 (3), in publication (2013).

32. L. COX, "LNK3DNT Geometry Support: User Guidance for Creating and Embedding," LA-UR-11-01655, Los Alamos National Laboratory (2011).

33. R. E. ALCOUFFE et al., "PARTISN: A Time-Dependant, Parallel Neutral Particle Transport Code System," LA-UR-08-07258, Los Alamos National Laboratory (2008).

34. DESSAULT SYSTEMS SIMULA CORP., Abaqus Users Manuals, Version 6.9, Providence, RI (2009).

35. R. L. MARTZ, "MCNP6 Unstructured Mesh Initial Validation and Performance Results," LA-UR-11-04657, Los Alamos National Laboratory (2011), Vol. 180, No. 3, pp. 316-336.

36. C. A. ANDERSON, K. C. KELLEY, and T. GOORLEY, "Mesh Human Phantoms with MCNP," LA-UR-12-00139, Los Alamos National Laboratory (2012).

37. J. BULL, "Magnetic Field Tracking Features in MCNP6," LA-UR-11-00872, Los Alamos National Laboratory (2011).

38. M. BERZ and K. MAKINO. "COSY INFINITY Version 8.1 User's Guide and Reference Manual," MSUHEP-20704, Michigan State University, East Lansing, MI (2002), http://cosy.pa.msu.edu.

39. J. S. HENDRICKS and B. QUITER, "MCNP/X Form Factor Upgrade for Improved Photon Transport," Nucl. Technol., 175, 150 (2011).

40. J. SWEEZY, "Pre-collision Next Event Estimator," LA-UR-11-01825, Los Alamos National Laboratory (2011).

41. R. PRAEL, "Tally Edits for the MCNP6 GENXS Option," LA-UR-11-02146, Los Alamos National Laboratory (2011).

42. M. Fensin, M. James, J. Hendricks, and T. Goorley, "The New MCNP6 Depletion Capability", LA-UR-11-07032, ICAPP, Chicago, June 2012.

43. McKinney, G. W., James, M. R., Armstrong, H. J., Clem, J. M., and Goldhagen, P., "MCNP6 Cosmic-Source Option," LA-UR-12-0196, Proceedings of the ANS Annual Meeting, Chicago, IL, June 24-28 (2012).

44. Palomares, J., and McKinney, G. W., "MCNP Simulations of Background Particle Fluxes from Galactic Cosmic Rays," LA-UR-13-20254, Proceedings of the ANS Annual Meeting, Atlanta, GA, June 16-20 (2013).

45. McKinney, G. W., "MCNP6 Enhancements of Delayed-Particle Production," LA-UR-11-6426, Proceedings of the 2012 IEEE NSS-MIC, Anaheim, CA, October 29 - November 3 (2012).

46. C. N. ZEEB and T. ZUKAITIS, "CTBS (Continuous Build and Test System) Poster," LA-UR 06-7356, Los Alamos National Laboratory (2006).

47. M. T. Sellers, T. Goorley, E.C. Corcoran, D.G. Kelly, "Modeling the Detection of Delayed Neutron Signatures in MCNP6 and Comparisons with Experimental 233U, 235U, and 239Pu Measurements", LA-UR-13-22525, Los Alamos National Laboratory, April 2013.

48. H. G. HUGHES, "Treating Electron Transport in MCNP," LA-UR-96-4583,

Los Alamos National Laboratory (1996); in: Proc. of the Training Course on the Use of MCNP in Radiation Protection and Dosimetry, Bologna, Italy, May 13-16, 1996, edited by G. GUALDRINI and L. CASALINI, ENEA, ISBN 88-8286-000-1.

49. K. KOBAYASHI, N. SUGIMURI, and Y. NAGAYA, "3-D Radiation Transport Benchmark Problems and Results for Simple 
Geometries with Void Regions," ISBN: 92-64-18274-8, NEA/OECD, Paris, France (2000).

50. Y. NAGAYA et al., "MVP/GMVP II : General Purpose Monte Carlo Codes for Neutron and Photon Transport Calculations based on Continuous Energy and Multigroup Methods," JAERI-1348, Japanese Atomic Energy Research Institue (2005).

51. S. G. MASHNIK, "Validation and Verification of MCNP6 Against Intermediate and High-Energy Experimental Data and Results by Other Codes," Eur. Phys. J. Plus, 126, 49 (2011); arXiv:1011.4978.

52. S. G. MASHNIK, "Validation and Verification of MCNP6 Against High-Energy Experimental Data and Calculations by Other Codes. I. The CEM Testing Primer," LA-UR-11-05129, Los Alamos National Laboratory (2011).

53. R. D. MOSTELLER, "Validation Suites for $\mathrm{MCNP}^{\mathrm{TM}}$," LA-UR-02-0878, Los Alamos National Laboratory (2002); Proc. of the American Nuclear Society Radiation Protection and Sheidling Division 12th Biennial Topical Meeting, Santa Fe, NM, April 14-17, 2002, CD ROM ISBN: 8-89448-66-5, American Nuclear Society, Inc., La Grange Park, IL 60526 USA (2002).

54. International Handbook of Evaluated Criticality Safety Benchmark Experiments, NEA/NSC/DOC(95)03, OECD Nuclear Energy Agency (2008).

55. R. D. MOSTELLER, "An Expanded Criticality Validation Suite for MCNP," LA-UR-10-06230, Los Alamos National Laboratory (2010)

56. R. D. MOSTElleR, F. B. BROWN, and B. C. KIEDROWSKI, "An Expanded Criticality Validation Suite for MCNP,” LA-UR-11-04170,

Los Alamos National Laboratory (2011), Proc. Int. Conf. on Nuclear Criticality, Edinburgh, Scotland, 19 - 22 September, 2011, http://www.informaglobalevents.com/event/icnc2011.

57. S. G. MASHNIK, "Validation and Verification of MCNP6 Against High-Energy Experimental Data and Calculations by Ohter Codes. II. The LAQGSM Testing Primer," LA-UR-11-05627, Los Alamos National Laboratory (2011).

58. R. D. MOSTELLER and B. C. KIEDROWSKI, "The Rossi Alpha Validation Suite for MCNP," LA-UR-11-04409, Los Alamos National Laboratory (2011), Proc. Int. Conf. on Nuclear Criticality, Edinburgh, Scotland, $19-22$ September, 2011, http://www.informaglobalevents.com/event/icnc2011.

59. J. A. BUCHOLZ and S. C. FRANKLE, "Improving the LLNL Pulsed Sphere Experiments Database and MCNP Models," LA-UR-03-0609, Los Alamos National Laboratory (2003), Transactions of the American Nuclear Society, 2003.

60. A. SOOD, R. A. FORSTER, and D. K. PARSONS, "Analytical Benchmark Test Set for Criticality Code Verification," Prog. Nucl. Energy, 42, 55 (2003).

61. S.C. van der Marck, "Benchmarking ENDF/B-VII.0," Nuclear Data Sheets 107:3061:3118 (2006). 